\title{
On the Behaviour of Weighted Permutation Entropy on Fractional Brownian Motion in the Univariate and Multivariate Setting
}

\author{
Marisa Mohr \\ Institute of Information Systems \\ University of Lübeck \\ 23562 Lübeck, Germany
}

\author{
Florian Wilhelm \\ Data Mangement and Analytics \\ inovex $\mathrm{GmbH}$ \\ 76131 Karlsruhe, Germany
}

\author{
Ralf Möller \\ Institute of Information Systems \\ University of Lübeck \\ 23562 Lübeck, Germany
}

\begin{abstract}
The estimation of the qualitative behaviour of fractional Brownian motion is an important topic for modelling real-world applications. Permutation entropy is a well-known approach to quantify the complexity of univariate time series in a scalar-valued representation. As an extension often used for outlier detection, weighted permutation entropy takes amplitudes within time series into account. As many-real world problems deal with multivariate time series, these measures need to be extended though. First, we introduce multivariate weighted permutation entropy, which is consistent with standard multivariate extensions of permutation entropy. Second, we investigate the behaviour of weighted permutation entropy on both univariate and multivariate fractional Brownian motion and show revealing results.
\end{abstract}

\section{Introduction}

Time series data are part of many real-world applications, such as forecasting weather and stock markets or analysing medical signals or industrial sensor data. Modelling of timedependent dynamical systems with the specific properties of long-range dependence, fractality, or self-similarity is commonly described by fractional Brownian motion (fBm). Its foundations are based on a strong theory and proved to be successfully applicable in many use-cases (Nourdin 2013; Rostek 2009; Doukhan, Oppenheim, and Taqqu 2002). For the investigation of the qualitative behaviour of $\mathrm{fBm}$, efficient mappings from a dynamical system to a set of scalarvalued representations or features that capture specific properties are usually considered. Entropies, a representation from information theory, are promising through an encoding that preserves information content (Amigó 2010). Permutation entropy (PE) is a robust, scalar-valued representation for measuring the degree of complexity of a time series by analysing the distribution of ordinal patterns, i.e., by analysing its up and downs (Bandt and Shiha 2007). While classical time series analysis focuses on the time series itself, a non-parametric transformation into a sequence of ordinal patterns can reveal underlying dynamics of the generating system to detect causal information (Traversaro et al. 2018). While the associated entropy is low for a deterministic time

Copyright @ 2021 by the authors. All rights reserved. series, it approaches its maximum value in case of uncorrelated randomness or correlated complexity in the time series.

In the univariate case, $\mathrm{PE}$ on $\mathrm{fBm}$ is well understood (Zunino et al. 2008; Sinn and Keller 2011). Not only PE, but also the distribution of ordinal patterns of certain lengths yield interesting parameter functions, e.g., for descriptive purposes of autocorrelation (Bandt and Shiha 2007). The limitation of $\mathrm{PE}$ is mainly the inability to distinguish between different patterns of a given motif in amplitudes. Fadlallah et al. (2013) introduce weighted permutation entropy (WPE), which takes into account patterns that differ in amplitudes by assigning weights to each extracted pattern.

Furthermore, in many fields of applications, multivariate measurements are performed such as in economic time series (Gil-Alana 2003), or functional Magnetic Resonance Imaging of several brain regions (Achard et al. 2008). Therefore, representations need to be extended. First, this paper contributes a definition of multivariate weighted permutation entropy (MWPE) that is consistent with canonical multivariate extensions of PE. Second, this paper investigates the behaviour of WPE on univariate and multivariate $\mathrm{fBm}$. We show that in case of ordinal patterns of order $d=2$ weighting has no effect, while in case of order $d=3$ certain ordinal patterns have greater impact on WPE.

\section{Related Work}

Bandt and Shiha (2007) and Zunino et al. (2008) contribute significantly to an understanding of the underlying behaviour of PE under known structures such as fBm. They investigate the distribution of ordinal patterns of different orders and, if possible, provide closed formulas for entropy calculation as well as specific characteristics and relationships that are used to prove the theorems in this work.

Since many real-world problems involve multivariate time series, Mohr, Finke, and Möller (2020) investigate the behaviour of $\mathrm{PE}$ on $\mathrm{fBm}$ in a multivariate setting as well as on multiple scales, but without considering effects of amplitudes. When introducing univariate WPE, Fadlallah et al. (2013) also evaluate their method on multivariate data, but calculate WPE from a univariate point of view per variable, followed by an analysis of correlations. In this sense, we introduce and appropriate definition of MWPE and analyse its behaviour in the special case of $\mathrm{fBm}$ or multivariate fractional Brownian motion (mfBm). 
(a) fBm with $H=0.1$

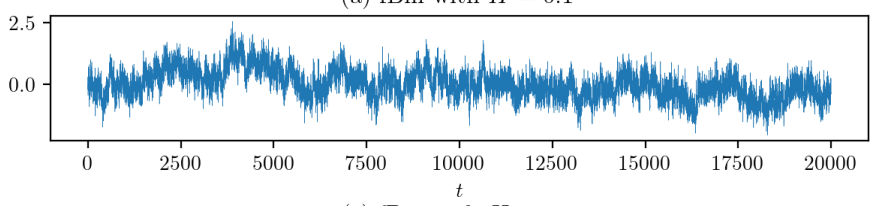

(c) fBm with $H=0.8$

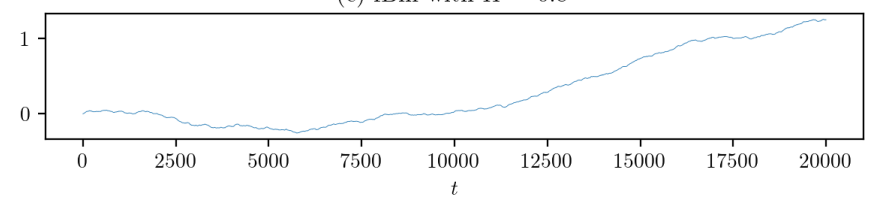

(b) fBm with $H=0.5$

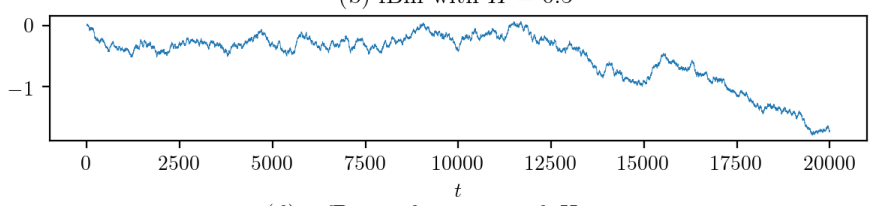

(d) $\mathrm{mfBm}$ with $m=4$ and $H_{i}=0.4$

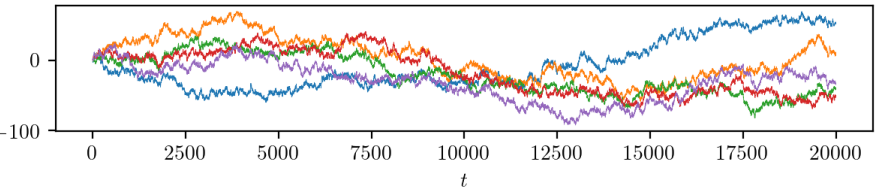

Figure 1: (a)-(c) Examples for fractional Brownian motion with different Hurst parameters $H$ and (d) multivariate fractional Brownian motion with variable-dimension $m=5$ and Hurst parameters $H_{i}=0.4$.

\section{Preliminaries}

We shortly formalise fractional Brownian motion and define key concepts of weighted permutation entropy.

\subsection{Multivariate Fractional Brownian Motion}

This work focuses on a class of special stochastic processes, namely the unique mean-zero Gaussian process, which is zero at origin and has stationary and self-similar increments, called fBm. A stochastic process, or more generally a mathematical object that is similar to itself at all scales, is called a fractal. When you zoom in on a fractal, it looks exactly like the original shape. The mathematical property is called selfsimilarity and is expressed by the so-called Hurst parameter $H \in(0,1)$. In case $H=1 / 2, \mathrm{fBm}$ corresponds to the ordinary Brownian motion. In case $H>1 / 2$, the process has a persistence property and positively correlated increments, i.e., an upward jump is more likely followed by another upward jump and vice versa. For $H \rightarrow 1$, the process becomes smoother, less irregular and more trendy. In case $H<1 / 2$, the process has negatively correlated increments and an antipersistence property. Figure 1 (a)-(c) shows three fBms with different Hurst parameters $H$.

Definition 1 (Multivariate fractional Brownian motion (Amblard et al. 2013)). An $m$-multivariate process $\left(\left(X^{i}(t)\right)_{i=1}^{m}\right)_{t \in \mathbb{R}}$ is called multivariate fractional Brownian motion (mfBm) with Hurst parameter $H=\left(H_{1}, \ldots, H_{m}\right)$, $H_{i} \in(0,1)$ for $i=1, \ldots, m$, and denoted as $\mathbf{B}_{H}^{m}(t)$, if it is

1. Gaussian distributed,

2. self-similar with Hurst parameter $H$, i.e.,

$$
\begin{array}{r}
\left(X_{1}(\alpha t), . ., X_{m}(\alpha t)\right)_{t \in \mathbb{R}} \\
\sim\left(\alpha^{H_{1}} X_{1}(t), . ., \alpha^{H_{m}} X_{m}(t)\right)_{t \in \mathbb{R}}
\end{array}
$$

for any $\alpha>0$, where $\sim$ denotes the equality of finitedimensional distributions and it has

3. stationary increments, i.e., $\mathbf{B}_{H}^{m}(t)-\mathbf{B}_{H}^{m}(s) \sim \mathbf{B}_{H}^{m}(t-s)$.

Multivariate self-similarity imposes many constraints on the covariance structure of $\mathrm{mfBm}$ (Lavancier, Philippe, and Surgailis 2009). The covariance structure is characterised by parameters $\sigma_{i}>0, \rho_{i j} \in(-1,1)$ and $\eta_{i j} \in \mathbb{R}$ for $i, j=1, \ldots, m$. Parameter $\sigma_{i}>0$ is the standard deviation of the $i$-th variable at time 1 . Parameter $\rho_{i j}=\rho_{j i}$ is the correlation coefficient between the variables $i$ and $j$ at time 1. Parameters $\eta_{i j}=-\eta_{j i}$ are antisymmetric and linked with the time-reversibility of $\mathrm{mfBm}$. Multivariate fractional Brownian motion can be characterised by its covariances and cross-covariances of its variables as follows.

Lemma 1 (Covariance Function of mfBm (Amblard and Coeurjolly 2011)). The $\mathrm{mfBm} \mathrm{B}_{H}^{m}(t)$ is marginally a $\mathrm{fBm}$, such that the covariance function of the $i$-th variable $B_{H_{i}}^{i}$ of $\mathrm{mBfm}$ is as in the univariate case

$\operatorname{Cov}\left(B_{H_{i}}^{i}(s), B_{H_{i}}^{i}(t)\right)=\frac{\sigma_{i}^{2}}{2}\left(|s|^{2 H_{i}}+|t|^{2 H_{i}}-|t-s|^{2 H_{i}}\right)$.

where $\sigma_{i}^{2}=\operatorname{Var}\left(B_{H_{i}}^{i}(1)\right)$. The cross-covariances of mfBm for all $(i, j) \in\{1, \ldots, m\}^{2}$ and $i \neq j$ are given by

$$
\begin{aligned}
\operatorname{Cov}\left(B_{H_{i}}^{i}(s), B_{H_{j}}^{j}(t)\right) \\
=\frac{\sigma_{i} \sigma_{j}}{2}\left(w_{i j}(-s)+w_{i j}(t)-w_{i j}(t-s)\right)
\end{aligned}
$$

where the function $w_{i j}(h)$ is defined as

$$
\begin{aligned}
& w_{i j}(h) \\
& = \begin{cases}\left(\rho_{i j}-\eta_{i j} \operatorname{sign}(h)\right)|h|^{H_{i}+H_{j}} & \text { if } H_{i}+H_{j} \neq 1, \\
\rho_{i j}|h|+\eta_{i j} h \log |h| & \text { if } H_{i}+H_{j}=1 .\end{cases}
\end{aligned}
$$

Figure 1 (d) shows an $\mathrm{mfBm}$ with $m=5$. For $m=1$, Definition 1 matches the univariate $\mathrm{fBm}$ denoted as $B_{H}(t)$. Moreover, setting $\rho_{i j}=1$ and $\eta_{i j}=0$ in Eqs. (2) and (3) is matching with the univariate case in Eq. (1).

\subsection{Ordinal Pattern Symbolisation}

For the investigation of the qualitative behaviour of $\mathrm{fBm}$ in this paper PE and WPE are used. To calculate the entropy of a time series it is necessary to encode the sequence of real-valued measurements into a sequence of symbols. As far as current research is concerned, there are two general approaches of symbolisation (Mohr et al. 2020). On the one hand, classical symbolisation approaches use threshold values and data range partitioning for symbol assignment, 


\section{$\circ \%$ \% \\ $(0,1,2),(2,1,0),(1,0,2),(2,0,1),(0,2,1),(1,2,0)$}

Figure 2: All possible ordinal patterns of order $d=3$.

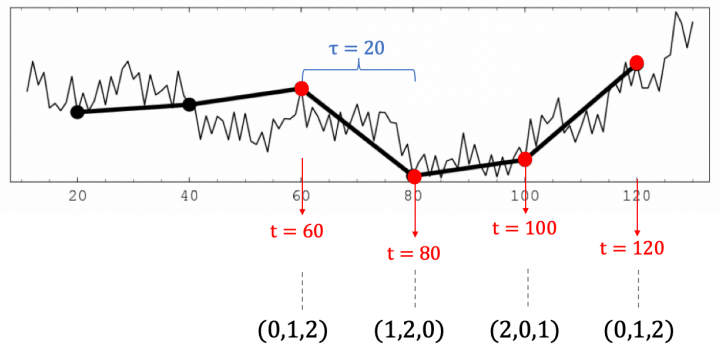

Figure 3: Ordinal pattern determination of order $d=3$ and time delay $\tau=20$ in a univariate time series.

such as the well-know Symbolic Aggregate ApproXimation (SAX) representation introduced by Chiu, Keogh, and Lonardi (2003). Ordinal pattern symbolisation is another approach based on an idea of Bandt and Pompe (2002) and uses the total order between two or more neighbours in a time series, encoded by permutations. The formalism and the advantages of the ordinal symbolisation scheme are introduced as follows. Compared to the previously used capitalisation $X_{i}$ for random variables in a dynamical system such as $\mathrm{fBm}$, we use a small notation $x_{i}$ for observations of random variables, also called paths.

Ordinal patterns describe the total order between two or more neighbours in a path, encoded by permutations.

Definition 2 (Univariate Ordinal Pattern). A vector $\left(x_{1}, \ldots, x_{d}\right) \in \mathbb{R}^{d}$ has ordinal pattern $\left(r_{1}, \ldots, r_{d}\right) \in \mathbb{N}^{d}$ of order $d \in \mathbb{N}$ if $x_{r_{1}} \geq \ldots \geq x_{r_{d}}$ and $r_{l-1}>r_{l}$ in the case $x_{r_{l-1}}=x_{r_{l}}$.

Note that equality of two values within a pattern is not allowed. In this case, for example, the newer value is replaced with a smaller value. Figure 2 shows all possible ordinal patterns of order $d=3$ of a vector $\left(x_{1}, x_{2}, x_{3}\right)$. To symbolise a time series $\left(x_{1}, x_{2}, \ldots, x_{T}\right) \in \mathbb{R}^{T}$ each point in time $t \in\{d, \ldots, T\}$ is assigned its ordinal pattern of order $d$. The order $d$ is chosen to be much smaller than the total length $T$ of the time series to look at smaller windows within the series and their distributions of up and down movements. To asses the overarching trend, the delayed behaviour is of interest. The time delay $\tau \in \mathbb{N}$ is the delay between successive points in the symbol sequences. Different delays show different details of the structure of a time series. Figure 3 visualises the ordinal pattern determination of order $d=3$ and time delay $\tau=20$ of four different time points in a univariate time series.

The ordinal approach has notable advantages in its practical application. First of all, the method is conceptually simple. Second, it is not necessary to have previous knowledge about the data range or type of time series. Third, the ordinal approach supports robust and fast implementations (Keller et al. 2017; Piek, Stolz, and Keller 2019). Fourth, it allows for an easier estimation of a good symbolisation scheme compared to the classical symbolisation approaches (Keller, Maksymenko, and Stolz 2015; Stolz and Keller 2017).

Not the ordinal patterns themselves, but their distributions in different parts of a univariate time series $\left(x_{t}\right)_{t=1}^{T}$ are of interest. Thus, each pattern is identified with exactly one of the ordinal pattern symbols $j=1,2, \ldots, d$ !. Using the distribution of the ordinal pattern symbols, its entropy can be calculated using the well-known formula of (Shannon) entropy resulting in the definition of permutation entropy:

Definition 3 (Permutation entropy (Bandt and Pompe 2002)). The permutation entropy (PE) of order $d \in \mathbb{N}$ and delay $\tau \in \mathbb{N}$ of a univariate time series $\mathbf{x}=\left(x_{t}\right)_{t=1}^{T}, T \in \mathbb{N}$ is defined by

$$
\mathrm{PE}_{d, \tau}(\mathbf{x})=-\sum_{j=1}^{d !} p_{j}^{\tau, d} \ln p_{j}^{\tau, d},
$$

where $p_{j}^{\tau, d}$ is the frequency of ordinal pattern $j$ in the time series.

Depending on the area of research, entropy is a measure for quantifying inhomogeneity, impurity, complexity and uncertainty or unpredictability. For time series with maximum random ordinal pattern (resulting in a uniform pattern distribution due to uniqueness), $\mathrm{PE}$ is $\ln (d !)$. For time series with regular pattern, PE is equal to zero (Amigó 2010).

\subsection{Weighted Permutation Entropy}

The main shortcoming in the above definition of PE is that no other information is preserved during the extraction of the ordinal patterns except for the order structure. Information about the amplitude in a time series is lost. However, ordinal patterns with large differences in amplitude should contribute in different ways to the calculation of PE. The weighted permutation entropy introduced by Fadlallah et al. (2013) allows for weighting of ordinal patterns by exploiting amplitude information resulting from small fluctuations in the time series due to the effect of noise to be weighted less than ordinal patterns with large amplitudes.

Definition 4 (Weighted permutation entropy (Fadlallah et al. 2013)). The weighted permutation entropy (WPE) of an univariate time series $\mathbf{x}=\left(x_{t}\right)_{t=1}^{T}, T \in \mathbb{N}$, with order $d \in \mathbb{N}$ and delay $\tau \in \mathbb{N}$ is defined as

$$
\mathrm{WPE}_{d, \tau}(\mathbf{x})=-\sum_{j}^{d !} w p_{j}^{\tau, d} \ln w p_{j}^{\tau, d}
$$

$$
\begin{aligned}
& \text { with } w p_{j}^{\tau, d} \\
& =\frac{\sum_{t \leq T} w_{t} \cdot\left[\left(x_{t-(d-1) \tau}, \ldots, x_{t-\tau}, x_{t}\right) \text { has pattern } j\right]}{\sum_{t \leq T} w_{t} \cdot\left[\left(x_{t-(d-1) \tau}, \ldots, x_{t-\tau}, x_{t}\right)\right]},
\end{aligned}
$$

where $w_{t}=\frac{1}{d} \sum_{k=1}^{d}\left(x_{t-(k-1) \tau}-\bar{x}_{t}^{d, \tau}\right)^{2}$ is the empirical variance of the sub-sequence and $\bar{x}_{t}^{d, \tau}$ denotes the arithmetic mean that is $\bar{x}_{t}^{d, \tau}=\frac{1}{d} \sum_{k=1}^{d} x_{t-(k-1) \tau}$ and $[x]=1$ if $x, 0$ otherwise. 


\section{Multivariate Weighted Permutation Entropy}

In this section we introduce a definition for multivariate weighted permutation entropy based on an unweighted canonical multivariate extension of PE introduced by Keller and Lauffer (1999) called pooled permutation entropy (PPE). The idea of PPE is to pool the frequencies of $d$ ! ordinal patterns with respect to all $m$ variables as input for multivariate entropy calculation. In addition to PPE and by analogy with WPE, in a canonical definition of multivariate weighted permutation entropy, the patterns have to be weighted before frequencies are determined.

More specifically, the determination of MWPE has to be carried out as follows. (I) For each variable $i=1, \ldots, m$ and for each ordinal pattern $j=1, \ldots, d$ ! select all time steps in the time series $t \in[d \tau-\tau+1, T]$, for which the variable time pair $(i, t)$ has the ordinal pattern $j$. (II) Add up the weights $w_{t}$, i.e., $\mathbf{w}_{\mathbf{i j}}=\sum_{t=d \tau-\tau+1}^{T} w_{t}$ for all selected ordinal pattern vectors $j$ and for each variable $i=1, \ldots, m$. Note that the total count of weights $w_{t}^{i}$ for each variable $i$ is $\delta:=T-(d \tau-\tau)$. (III) Divide the weighted sum $\mathbf{w}_{\mathbf{i j}}$ by the total sum of all $m \cdot \delta$ weights to obtain the weighted frequencies for every pattern $j$. (IV) Store the weighted frequencies in a weighted pooling matrix $P_{w}^{\tau, d} \in \mathbb{R}^{m \times d}$ ! , which reflects the weighted distribution of the ordinal patterns in the multivariate time series across its $m$ variables. Based on the weighted pooling matrix $P_{w}^{\tau, d}$, the multivariate weighted permutation entropy can be calculated as follows.

Definition 5 (Multivariate weighted permutation entropy). The multivariate weighted permutation entropy (MWPE) of a multivariate time series $\mathbf{X}=\left(\left(x_{t}^{i}\right)_{i=1}^{m}\right)_{t=1}^{T}$ is defined as $\mathrm{PE}$ of the marginal weighted frequencies $P_{w, j}^{\tau, d}=\sum_{i=1}^{m} P_{w_{i j}}^{\tau, d}$ for $j=1, \ldots, d$ ! describing the distribution of the weighted ordinal pattern and can be calculated as

$$
\mathrm{MWPE}_{d, \tau}(\mathbf{X})=-\sum_{j}^{d !} P_{w \cdot j}^{\tau, d} \ln P_{w \cdot j}^{\tau, d} .
$$

\section{Weighted Permutation Entropy Applied to Fractional Brownian Motion}

In the following, we investigate the behaviour of WPE on $\mathrm{fBm}$ as well as MWPE on $\mathrm{mfBm}$ in theoretical terms. For this purpose, we use univariate results by Bandt and Shiha (2007), who show that first, for fBms the ordinal patterns of order $d=2$ are equally distributed, more specifically

$$
p_{01}^{\tau}=p_{10}^{\tau}=1 / 2
$$

for all $\tau$. Second, the distribution of ordinal patterns of order $d=3$ of univariate $\mathrm{fBms}$ is given by

$$
p_{012}^{\tau}=1 / \pi \arcsin 2^{H-1}:=u
$$

for all $\tau$. Then, for all $\tau$ it is

$$
p_{j}^{\tau}= \begin{cases}u & \text { if } j=(0,1,2),(2,1,0), \\ (1-2 u) / 4 & \text { otherwise. }\end{cases}
$$

The distribution of ordinal patterns of order $d=4$ can also be expressed in a more complex formula, which can be looked up in the work of Bandt and Shiha (2007). For order $d>4$, there are no closed formulas.

\subsection{The Univariate Case}

We first consider the behaviour of WPE of order $d=2$ on $\mathrm{fBm}$ before transferring theory to patterns of order $d=3$.

Theorem 1. The WPE of order $d=2$ for all delays $\tau \in \mathbb{N}$ on $\mathrm{fBm} B_{H}(t)$ is given by

$$
\operatorname{WPE}\left(B_{H}(t)\right)=-\ln (1 / 2) .
$$

Proof. WPE differs from PE in that the ordinal patterns are weighted depending on their position $t$ according to Eq. (6). For a weight $w_{t}$ of order $d=2$, i.e., of two time steps $x_{t-1}$ and $x_{t}$, we have

$$
\begin{aligned}
w_{t} & =\frac{1}{2} \sum_{k=1}^{2}\left(x_{t-(k-1) \tau}-\bar{x}_{t}^{2, \tau}\right)^{2} \\
& =\frac{1}{2}\left(x_{t}-x_{t-\tau}\right)^{2} .
\end{aligned}
$$

Since $x_{t} \sim B_{H}(t)$, we conclude from Definition 1, property (3), i.e., fBm has stationary increments, that

$$
\frac{1}{2}\left(B_{H}(t)-B_{H}(t-\tau)\right)^{2} \sim \frac{1}{2}\left(B_{H}(\tau)\right)^{2}
$$

with $\operatorname{Var}\left(B_{H}(\tau)\right)=\sigma^{2} \tau^{2 H}$ and $\sigma^{2}=\operatorname{Var}\left(B_{H}(1)\right)=s^{2 H}$ as in Eq. (1). Consequently, the weights $w_{t}$ are independently distributed from $t$, i.e.,

$$
w_{t} \sim \mathcal{N}\left(0, \frac{1}{2}(s \tau)^{2 H}\right) .
$$

Considering the distribution of all possible realisations of $\mathrm{fBm}$, we see from the use of the weights $w_{t}$ from Eq. (6) in the calculation of WPE that it cancels out for a constant delay $\tau \in \mathbb{N}$.

With Eq. (8) follows $\operatorname{WPE}\left(B_{H}(t)\right)=-\ln (1 / 2)$.

Note that in the case of $d=3$, however, the weighting has influence due to the lack of symmetry. Moreover, the weight of order $d=3$ is given by

$$
\begin{aligned}
w_{t}= & \frac{2}{9}\left(x_{t}^{2}+x_{t-1}^{2}+x_{t-2}^{2}\right. \\
& \left.-x_{t} x_{t-1}-x_{t} x_{t-2}-x_{t-1} x_{t-2}\right) \\
= & \frac{2}{9}\left(x_{t}^{2}-x_{t}\left(x_{t-1}-x_{t-2}\right)\right. \\
& \left.\left.+x_{t-1}^{2}+x_{t-2}^{2}-x_{t-1} x_{t-2}\right)\right)
\end{aligned}
$$

For example, in case of $0<x_{t-2}<x_{t}^{\prime}<x_{t-1}<x_{t}$, it is

$$
{x^{\prime}}_{t}^{2}-x_{t}^{\prime}\left(x_{t-1}-x_{t-2}\right)<x_{t}^{2}-x_{t}\left(x_{t-1}-x_{t-2}\right) .
$$

It follows $w_{x_{t}^{\prime}}<w_{x_{t}}$. Consequently, it is more likely that the ordinal patterns $(0,1,2)$ and $(2,1,0)$ have higher weights than the other four ordinal pattern. For $H>0.5$, fBm is positively correlated, i.e., after an upward jump a further upward jump is more likely to follow and vice versa, which increases the effect. With Eq. (10), WPE decreases faster than PE. For ordinal patterns with $d>3$ analogous behaviour results. We confirm this theory in an experimental evaluation in the next section. 
(a) PE and WPE on $\mathrm{fBm}$
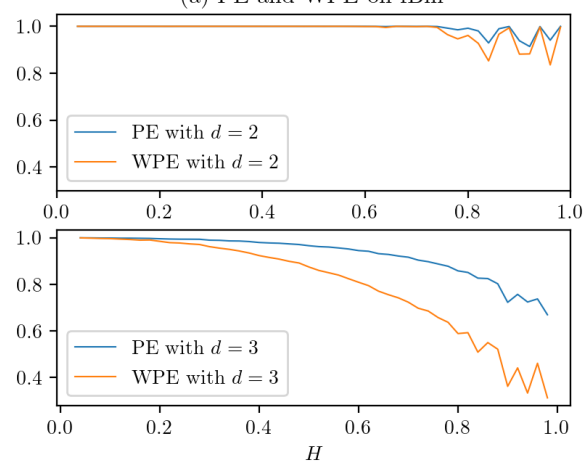

(b) PPE and MWPE on 3-fBm

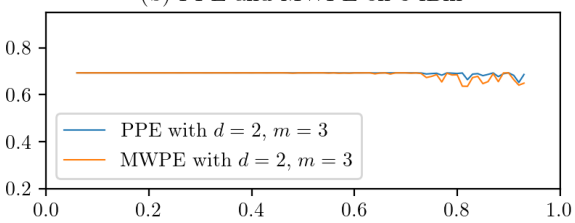

(c) PPE and MWPE on 5-fBm

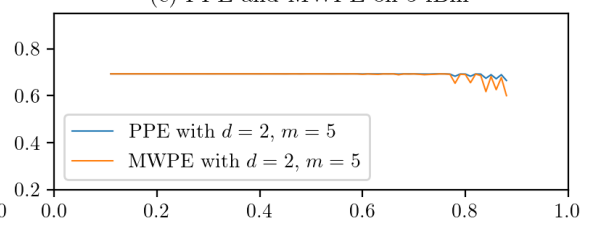

Figure 4: Comparison of permutation entropy and weighed permutation entropy of order $d=2$ and $d=3$ on fractional Brownian motion in relation to Hurst parameter $H$ in univariate case as well as multivariate case.

\subsection{The Multivariate Case}

Again, we first consider the behaviour of MWPE of order $d=2$ on $\mathrm{mfBm}$ before transferring the theory to patterns of order $d=3$.

Theorem 2. The MWPE of order $d=2$ for all delays $\tau \in \mathbb{N}$ on $m f B m \mathbf{B}_{H}^{m}(t)$ is given by

$$
\operatorname{MWPE}\left(\mathbf{B}_{H}^{m}(t)\right)=-\ln (1 / 2)
$$

for all Hurst parameters $H_{i}$ and variable-dimensions $m$.

Proof. As in Definition 5, the calculation of MWPE is based on the sum of weights $\mathbf{w}_{\mathbf{i j}}$ for a ordinal pattern $j$ and for each variable $i=1, \ldots, m$. The arguments from the proof of Theorem 1 can be adopted directly. Calculating the frequencies of ordinal patterns for the weighted pooling matrix $P_{w}^{\tau, d} \in \mathbb{R}^{m \times d !}$ results in a equal distribution with

$$
P_{w_{i,(1,0)}}^{\tau, 2}=P_{w_{i,(0,1)}}^{\tau, 2}=\frac{1}{2 m}
$$

for all $i=1, . ., m$. By calculating the marginal relative frequencies, the number of variables $m$ is reduced, i.e.,

$$
P_{w \cdot j}^{\tau, 2}=\frac{1}{2}
$$

for $j=\{(0,1),(1,0)\}$. It follows

$$
\operatorname{MWPE}\left(\mathbf{B}_{H}^{m}(t)\right)=-\sum_{j}^{2} P_{w \cdot j}^{\tau, 2} \ln P_{w \cdot j}^{\tau, 2}=-\ln (1 / 2) .
$$

In summary, MWPE of order $d=2$ does not depend on the delay $\tau$, the Hurst parameter $H$ or on the number of variables $m$, nor does the weighting have any influence on the calculation of MWPE.

Note that as in the univariate case with $d=3$, weighting has an impact due to lack of symmetry. The weights for strictly ascending and descending ordinal patterns $(0,1,2)$ and $(2,1,0)$, respectively, are likely to be higher than others. Analogous to Eqs. (8) and (10), Mohr, Finke, and Möller
(2020) show that the behaviour of PPE of order $d=3$ on $\mathrm{mfBm}$ depends monotonically on the Hurst parameter $H \in \mathbb{R}^{m}$. The calculation of the weights as well as the distribution of the ordinal patterns lead to an increased influence of certain patterns in the calculation of MWPE. In particular, if $H>0.5$, i.e., $\mathrm{fBm}$ is positively correlated, the effect is again amplified. MWPE decreases faster than PPE. We confirm this theory in an experimental evaluation in the next section.

\section{Experimental Evaluation}

We investigate the behaviour of WPE or MWPE on $\mathrm{ABm}$ or $\mathrm{mfBm}$ with respect to its Hurst parameter in an experimental evaluation. In the univariate case, all experimental calculations are based on a simulation of fBm using the fbm package available on PyPI. In the multivariate case, all experimental calculations are based on a simulation of $\mathrm{mfBm}$ an algorithm implemented by Amblard et al. (2013) with a parameter setting of $\rho_{i, j}=0.3$, and $\eta_{i, j}=0.1 /\left(1-H_{i}-H_{j}\right)$ (see Lemma 1). The lengths $T=20000$ of $\mathrm{fBms}$ and $T=3500$ of $\mathrm{mfBms}$ are assumed to be large. For a small length $T$ the estimates of the probabilities for the ordinal patterns differ from the true values of a hypothetical time series of infinite length.

As in the upper part of Figure 4(a), WPE of order $d=2$ on $\mathrm{fBm}$ behaves like PE (normalised in both cases), namely constant due to the equal distribution of ordinal patterns. This result confirms Theorem 1. As in the lower part of Figure 4(a), WPE of order $d=3$ decreases with increasing Hurst parameter $H$, faster than PE, and also over a larger data range, resulting from the fact that some of the patterns (specifically $(0,1,2)$ and $(2,1,0))$ have a greater impact than the others. As in the upper part of Figure 4(b) and (c), MWPE of order $d=2$ behaves like PPE on $\mathrm{mfBm}$, independent of the number of variable dimensions $m$. In particular, MWPE is constant $-\ln (1 / 2)$ confirming Theorem 2 . As in the lower part of Figure 4(b) and (c), MWPE of order $d=3$ decreases faster than PPE, with increasing Hurst parameters $H_{i}=H_{j}$ for all $i=j$. This confirms once again that the strictly ascending or descending patterns have a greater im- 
pact on the calculation of MWPE than with PPE.

In all sub-figures, larger deviations from the theoretical value can be seen starting at a Hurst parameter of about $H>0.75$. As discussed by Dávalos et al.; Mohr, Finke, and Möller (2018; 2020), the larger deviations are due to the experimental length limitation. In the lower part of Figure 4(c) the number of variable dimensions $m$ contributes to a better estimation of the MWPE due to the increasing number of ordinal patterns, so that the deviations become smaller. Thus, the experimental results underpin our theoretical findings.

\section{Conclusion and Future Work}

In this paper, we proposed a well-defined definition of MWPE, that is consistent to standard multivariate extensions of PE. In addition, we presented a theoretical as well as an experimental analysis of the behaviour of WPE on $\mathrm{fBm}$ and MWPE on mfBm. In case of order $d=2$, we have shown that WPE and MWPE match with PE and PPE. There is no effect of weighting. In case of order $d=3$, we have shown that strictly ascending or strictly descending ordinal patterns $(0,1,2)$ or $(2,1,0)$ have greater weights and thus a greater impact on WPE and MWPE than the other four possible ordinal patterns. As a result, WPE and MWPE of order $d=3$ decrease faster than PE and PPE with increasing Hurst parameter $H$, and over a larger normalised data range.

The theoretical results provided in this work can simplify the estimation of the Hurst parameter due to a more powerful representation of WPE and MWPE in contrast to the unweighted variants. Classical data analysis and machine learning tasks, such as outlier detection or time series classification, can also benefit. However, to confirm the overall effectiveness and efficiency of WPE and MWPE of order $d=3$, especially on other signals from real world applications, further analyses and tests are necessary. Furthermore, the canonical definition of MWPE does not take into account the interaction of different Hurst parameters $H_{i}$ in form of cross-correlations from Eqs. (2) and (3). In this case, it may be useful to consider further multivariate versions of PE using principle component analysis as proposed by Mohr et al. (2020).

\section{References}

Achard, S.; Bassett, D. S.; Meyer-Lindenberg, A.; and Bullmore, E. 2008. Fractal connectivity of long-memory networks. Physical Review E 77(3).

Amblard, P.-O., and Coeurjolly, J.-F. 2011. Identification of the Multivariate Fractional Brownian Motion. IEEE Transactions on Signal Processing 59(11):5152-5168.

Amblard, P.-O.; Coeurjolly, J.-F.; Lavancier, F.; and Philippe, A. 2013. Basic properties of the Multivariate Fractional Brownian Motion. Séminaires et congrès 28:65-87.

Amigó, J. 2010. Permutation Complexity in Dynamical Systems: Ordinal Patterns, Permutation Entropy and All That. Springer Series in Synergetics. Berlin Heidelberg: Springer-Verlag.

Bandt, C., and Pompe, B. 2002. Permutation Entropy: A Natural Complexity Measure for Time Series. Physical Review Letters 88(17):174102. Publisher: American Physical Society.

Bandt, C., and Shiha, F. 2007. Order Patterns in Time Series. Journal of Time Series Analysis 28(5):646-665.
Chiu, B.; Keogh, E.; and Lonardi, S. 2003. Probabilistic Discovery of Time Series Motifs. In Proceedings of the Ninth ACM SIGKDD International Conference on Knowledge Discovery and Data Mining, KDD '03, 493-498. ACM.

Doukhan, P.; Oppenheim, G.; and Taqqu, M. 2002. Theory and Applications of Long-Range Dependence. Springer Science \& Business Media.

Dávalos, A.; Jabloun, M.; Ravier, P.; and Buttelli, O. 2018. Theoretical Study of Multiscale Permutation Entropy on Finite-Length Fractional Gaussian Noise. In 2018 26th European Signal Processing Conference (EUSIPCO), 1087-1091. ISSN: 2076-1465.

Fadlallah, B.; Chen, B.; Keil, A.; and Principe, J. 2013. Weightedpermutation entropy: A complexity measure for time series incorporating amplitude information. Physical review. E, Statistical, nonlinear, and soft matter physics 87:022911.

Gil-Alana, L. A. 2003. A fractional multivariate long memory model for the US and the Canadian real output. Economics Letters 81(3):355-359.

Keller, K., and Lauffer, H. 1999. Symbolic analysis of highdimensional time series. In Int. J. Bifurcation Chaos, 2657-2668.

Keller, K.; Mangold, T.; Stolz, I.; and Werner, J. 2017. Permutation Entropy: New Ideas and Challenges. Entropy 19(3):134.

Keller, K.; Maksymenko, S.; and Stolz, I. 2015. Entropy determination based on the ordinal structure of a dynamical system. Discrete and Continuous Dynamical Systems - Series B 20(10):35073524.

Lavancier, F.; Philippe, A.; and Surgailis, D. 2009. Covariance function of vector self-similar processes. Statistics \& Probability Letters 79(23):2415-2421.

Mohr, M.; Wilhelm, F.; Hartwig, M.; Möller, R.; and Keller, K. 2020. New approaches in ordinal pattern representations for multivariate time series. Proceedings of the 33rd International Florida Artificial Intelligence Research Society Conference (FLAIRS-33) 124-129.

Mohr, M.; Finke, N.; and Möller, R. 2020. On the Behaviour of Permutation Entropy on Fractional Brownian Motion in a Multivariate Setting. In Proceedings of the Asia-Pacific Signal and Information Processing Association Annual Summit and Conference 2020 (APSIPA-ASC).

Nourdin, I. 2013. Selected Aspects of Fractional Brownian Motion. Springer Science \& Business Media.

Piek, A. B.; Stolz, I.; and Keller, K. 2019. Algorithmics, Possibilities and Limits of Ordinal Pattern Based Entropies. Entropy 21(6):547.

Rostek, S. 2009. Option Pricing in Fractional Brownian Markets. Springer Science \& Business Media.

Sinn, M., and Keller, K. 2011. Estimation of ordinal pattern probabilities in gaussian processes with stationary increments. Comput. Stat. Data Anal. 55(4):1781-1790.

Stolz, I., and Keller, K. 2017. A General Symbolic Approach to Kolmogorov-Sinai Entropy. Entropy 19(12):675.

Traversaro, F.; Redelico, F. O.; Risk, M. R.; Frery, A. C.; and Rosso, O. A. 2018. Bandt-Pompe symbolization dynamics for time series with tied values: A data-driven approach. Chaos: An Interdisciplinary Journal of Nonlinear Science 28(7).

Zunino, L.; Pérez, D. G.; Martín, M. T.; Garavaglia, M.; Plastino, A.; and Rosso, O. A. 2008. Permutation entropy of fractional Brownian motion and fractional Gaussian noise. Physics Letters A 372(27):4768-4774. 